\title{
Assessment of insulin-like growth factor 1 receptor as an oncogene in esophageal squamous cell carcinoma and its potential implication in chemotherapy
}

\author{
WANG MA ${ }^{1 *}$, TENGFEI ZHANG ${ }^{1,2^{*}}$, JIAN PAN $^{3}$, NI SHI $^{4}$, QINGXIA FAN ${ }^{1}$, \\ LIUXING WANG ${ }^{1}$ and SHIH HSIN LU ${ }^{1,5}$
}

${ }^{1}$ Department of Oncology, ${ }^{2}$ Biotherapy Center, The First Affiliated Hospital of Zhengzhou University, Zhengzhou, Henan;
${ }^{3}$ Department of Hematology and Oncology, Children's Hospital of Soochow University, Suzhou, Jiangsu, P.R. China;
${ }^{4}$ Department of Internal Medicine, The Ohio State University, Columbus, OH, USA; ${ }^{5}$ State Key Laboratory of
Molecular Oncology, Department of Etiology and Carcinogenesis, Cancer Institute and Hospital,
Chinese Academy of Medical Sciences and Peking Union Medical College, Beijing, P.R. China

Received March 26, 2014; Accepted June 27, 2014

DOI: $10.3892 /$ or.2014.3348

\begin{abstract}
Insulin-like growth factor-1 receptor (IGF-1R) is a tyrosine kinase receptor implicated in the pathogenesis of multiple cancers. After ligand binding, IGF-1R can initiate the activation of the PI3K/AKT/mTOR and Ras/Raf/MEK/ MAPK pathways to modulate cell proliferation, survival, differentiation, motility, invasion and angiogenesis. IGF-1R is a prerequisite for tumor progression and is one of the most attractive targets for therapeutic interventions in several types of cancer. In the present study, we determined the expression of IGF-1R in an esophageal squamous cell carcinoma (ESCC) cohort, investigated the detailed function of IGF-1R and screened the potential application of IGF-1R in the clinic. We verified the higher expression of IGF-1R in ESCC tumor tissues as compared to adjacent normal tissues. We also found that high expression of IGF-1R was associated with advanced tumor progression. We used ESCC cell lines and a mouse xenograft model to detect the function of IGF-1R in vitro and in vivo. Our results suggest the oncogenic function of IGF-1R in regulating cell proliferation, clonogenesis, the cell cycle and apoptosis. In addition, we found that IGF-1R was associated with the response to standard chemotherapy drugs 5-FU and cisplatin in an ESCC cell line. More importantly, we confirmed that the serum concentration of IGF-1/IGFBP3 can be used for predicting response to chemotherapy, and increased serum levels of IGF-1 and IGFBP-3 are associated with significantly
\end{abstract}

Correspondence to: Professor Shih Hsin Lu, State Key Laboratory of Molecular Oncology, Department of Etiology and Carcinogenesis, Cancer Institute and Hospital, Chinese Academy of Medical Sciences and Peking Union Medical College, Beijing, P.R. China

E-mail: shlu1212@gmail.com

*Contributed equally

Key words: IGF-1R, ESCC, oncogene, chemotherapy response higher rates of tumor response. In the present study, we demonstrated that IGF-1R is an important oncogene in ESCC and can be used to detect the chemotherapeutic response.

\section{Introduction}

Esophageal cancer is the eighth most common cancer worldwide with distinct geographical and ethnic characteristics (1). In China, it ranks as the fourth leading cause of cancer related mortality (2). Esophageal squamous cell carcinoma (ESCC) is the dominant type of esophageal malignancy in China. ESCC carcinogenesis is a multistage process characterized by morphological changes from normal esophagus to basal cell hyperplasia, dysplasia, carcinoma in situ and squamous cell carcinoma (SCC). It involves complex interactions between the environment and genetic factors (3). Although early intervention, as well as surgical, medical and radiotherapy techniques have undergone great achievements to date, the 5-year survival rate of ESCC patients remains low $(4,5)$. Since most patients are at an advanced stage at initial diagnosis, the chance of surgery is lost (6). Chemotherapy-based comprehensive treatment is the main treatment for advanced patients. Yet, chemotherapeutic outcomes are not optimistic due to the poor selection and serious adverse reactions of standard chemotherapy drugs. Therefore, targeted therapy drugs that can avoid the chemotherapy-induced adverse reactions and elevate the treatment effect are critical to reduce the morbidity and mortality of ESCC.

Insulin-like growth factor-1 receptor (IGF-1R) is a tyrosine kinase receptor implicated in the pathogenesis of multiple cancers (7). Overexpression of IGF-IR has been reported in a range of human solid tumors such as breast, non-small cell lung and prostate cancer, sarcomas, hepatocellular carcinoma and pancreatic, ovarian and gastrointestinal cancers (8-11). After ligand binding, IGF-1R can initiate the activation of the PI3K/AKT/mTOR signaling and Ras/Raf/MEK/MAPK pathways resulting in the activation of multiple transcription factors such as ELK-1, CREB and AP-1 to modulate cell 
proliferation, survival, differentiation, motility, invasion and angiogenesis $(12,13)$. Furthermore, more and more evidence indicates that IGF-1R is a prerequisite for tumor progression and is involved in the critical steps of the metastatic cascade $(14,15)$. Therefore IGF-1R is one of the most attractive targets for cancer therapeutic interventions (16).

In addition to IGF-1R, the IGF signaling system consists of another two ligands, IGF-1 and IGF-II; another two cell membrane receptors, IGF-IIR and insulin receptor; and six IGF binding proteins (IGFBP 1-6) (17). High circulating levels of IGF-I and a decreased level of IGFBP3 have been linked to increased cancer risk and have been identified as prognostic markers for various cancers such as breast, prostate, colon and lung cancer (18). Almost $90 \%$ of the circulating IGFs bind to IGFBP-3. IGFBP-3 inhibits IGF action at the cellular level by competitively binding IGFs and thereby preventing their binding to the IGF-1R. The molar ratio of IGF-I to IGFBP-3 has been regarded as an important index of IGF-I bioavailability. IGF-1R is associated with the sensitivity to chemotherapy in several types of cancers including lung, colon and head and neck cancer (19-21). The serum concentrations of IGF1 and IGFBP-3 may also play a role in the response to the chemotherapies.

IGF-1R has been reported as a marker for prognosis and as a therapeutic target in human ESCC (22). IGF-I autocrine system could stimulate tumor growth and chemoresistance in human esophageal carcinoma cells (23). However, the detailed biological function of IGF-1R in ESCC still requires investigation, and the implications of IGF-1R in treatment are still unclear. In this study, we determined the expression of IGF-1R in ESCC patients and investigated the biological function of IGF-1R in ESCC cell lines in a xenograft animal model. The overall objective of this study was to identify the potential application of IGF-1R in ESCC clinical treatment.

\section{Materials and methods}

Ethics statement. This study was approved by the Institutional Review Boards of the First Affiliated Hospital of Zhengzhou University. Written informed consent was obtained from all participants for the use of their blood or tissue samples. Full consent was obtained from the Institutional Review Boards of the First Affiliated hospital of Zhengzhou University. Animal care and experiments were approved by the Institute Animal Care and Use Committee of the Cancer Institute and Hospital, Chinese Academy of Medical Sciences and Peking Union Medical College.

Human ESCC cell lines and ESCC patient samples. ESCC cell lines EC9706, EC109 and NEC were gifts from the Department of Etiology and Carcinogenesis, Cancer Institute and Hospital, Chinese Academy of Medical Sciences and Peking Union Medical College. All the cells were cultured in RMPI-1640 medium with $10 \%$ fetal bovine serum (FBS), in $5 \% \mathrm{CO}_{2}$ at $37^{\circ} \mathrm{C}$.

Eighty human ESCC tissues used for immunohistochemical staining were collected from the First Affiliated Hospital of Zhengzhou University with the exact post-surgical pathological examination. Eighteen normal tumor-adjacent tissues were acquired from these patients at least $3 \mathrm{~cm}$ away from the primary tumor. These patients consisted of 52 females and 28 males. The mean age was 60 years. According to histological grade, 22 patients were in grade I, 37 patients were in grade II, and 21 patients were in grade III. According to clinical stage 6 patients were in stage I, 35 patients were in stage II, 32 patients were in stage III and 7 patients were in stage IV. There were 26 patients without lymph node metastasis and 53 patients with lymph node metastasis.

Serum samples used for the ELISA study were collected from 120 patients. These patients were diagnosed with ESCC between 2007 to 2009 at The First Affiliated Hospital of Zhengzhou University, Henan Cancer Hospital and Zhengzhou Central Hospital. None of these patients received any therapy before the two courses of chemotherapy treatment. The pre-therapy serum was collected 1-3 days before treatment, and the post-therapy serum was collected at the end of the treatments. These patients consisted of 51 females and 121 males. The mean age was 60 years. According to clinical stage, 43 patients were in stage IIB, 70 patients were in stage III and 59 patients were in stage IV. The chemotherapy effect was divided into 4 grades according to the Response Evaluation Criteria in Solid Tumors (RECIST): complete response (CR), all lesions disappeared lasting for at least 4 weeks; partial response (PR), the lesion volume shrank $>50 \%$ lasting for at least 4 weeks; stable disease (SD), no significant change, shrinking $<50 \%$ and enlargement $<25 \%$; progressive disease (PD), the lesion enlarged $>25 \%$.

Bioinformatic analysis. The microarray data GSE 33426 and GSE 23400 were downloaded from the GEO database: (http://www.ncbi.nlm.nih.gov/gds/). The differentially expressed genes were identified using GEO2R tools on GEO. The heatmap was generated using Genesis 1.0 (Graz, Austria). The biofunction and pathway analysis was performed with IPA $^{\circledR}$ Software (http://www.ingenuity.com).

Immunohistochemistry. Fresh tumor tissues were fixed with formalin, embedded and finally cut into $4-\mu \mathrm{m}$ sections. The sections were deparaffinized by dimethylbenzene and graded ethanol. Then the sections were incubated with $3 \% \mathrm{H}_{2} \mathrm{O}_{2}$, goat serum, anti-IGF-1R antibody, biotin-labeled secondary antibody, peroxidaze-conjugated streptavidin in order before DAB staining. All the reagents were purchased from ZSGB-Bio (Beijing, China). The staining intensity of IGF-1R was quantified into 3 groups according to the percentage of stained cells: negative, $0-5 \%$ cells were stained; weak positive, $>5-50 \%$ cells were stained; strong positive, $>50-100 \%$ cells were stained.

Western blot analysis. Protein was extracted from the cells using lysis buffer (50 mM Tris- $\mathrm{HCl} \mathrm{pH} 7.4,150 \mathrm{mM} \mathrm{NaCl}$, $1 \mathrm{mM}$ EDTA, $1 \%$ Triton X-100). The protein concentration was measured using the the Bradford method. The protein was separated by SDS-PAGE and transferred to a polyvinylidene fluoride (PVDF) membrane (Millipore, Billerica, MA, USA). After incubation with $10 \%$ milk, the primary IGF-1R antibody, and the secondary antibody, the blot signals were detected by chemiluminescence using the ECL kit (Applygen, Beijing, China). All the antibodies were purchased from Santa Cruz (Dallas, TX, USA). 
Stable IGF-1R-knockdown cell line. pGCsilencer ${ }^{\mathrm{TM}}$ U6/Neo/GFP/RNAi-IGF-1R plasmid used for the knockdown of IGF-1R and the pGCsilencer ${ }^{\mathrm{TM}} \mathrm{U6} / \mathrm{Neo} / \mathrm{GFP}$ negative control were obtained from Genechem Co. (Shanghai, China). Both plasmids were transfected into EC9706 cells using Lipofectamine $^{\mathrm{TM}} 2000$ (Invitrogen, Carlsbad, CA, USA). The cells were digested and seeded into a new 24-well plate, diluted 5 times, $24 \mathrm{~h}$ after the transfection. Then, the regular medium was changed to medium containing $600 \mu \mathrm{g} / \mathrm{ml} \mathrm{G} 418$ for stable transfection colony screening. After all of the cells without transfection died 4 days later, the remained cells were sustained by medium containing $200 \mu \mathrm{g} / \mathrm{ml} \mathrm{G} 418$ and accepted as the stable transfection cell lines: IGF-1R-knockdown cell line and blank transfection control cell line. Western blotting and RT-PCR verified the knockdown of IGF-1R in the cell lines.

Cell proliferation study. Cells were seeded in a 96-well plate at a density of 4,000 cells/well. The next day, $10 \mu 1 \mathrm{of} 5 \mathrm{mg} /$ $\mathrm{ml}$ MTT was added to the medium for a 3-h incubation, and the time point was set as zero then detected every $24 \mathrm{~h}$ for 6 days. Then the medium was removed, and $150 \mu 1$ DMSO was added. Finally the OD value was read at $490 \mathrm{~nm}$ on a Model 680 microplate reader (Bio-Rad, Hercules, CA, USA) after a 10-min incubation.

Flow cytometry. Cells were digested by trypsin, washed twice in cold PBS, fixed with ice cold $70 \%$ methanol, and incubated at $4^{\circ} \mathrm{C}$ overnight. Cells were then washed in PBS and incubated with $25 \mu \mathrm{g} / \mathrm{ml}$ propidium iodide containing $30 \mu \mathrm{g} / \mathrm{ml}$ ribonuclease for $30 \mathrm{~min}$ at room temperature. Cells were analyzed on the FACSCalibur Cell Sorting System (Becton Dickinson, Franklin Lakes, NJ, USA) using BD FACSuite ${ }^{\mathrm{TM}}$ Software in triplicate.

Cell colony formation study. Cells were seeded onto 6-well plates at a density of 300 cells/well. Following 12 days, visible colonies were fixed with methanol and stained with $0.4 \%$ crystal violet solution. Colonies were counted, and the number of visible colonies in each well was determined. Each assay was performed in triplicate.

Mouse ESCC xenograft study. Male BALB/c nude mice 5-6 weeks old were purchased from the Institute of Laboratory Animal Sciences, Chinese Academy of Medical Sciences and Peking Union Medical College. The mouse were divided into three groups and were inoculated with stable IGF-1R-knockdown cells, empty vector control cells or wild-type EC9706 cells, respectively (six mice in each group). A total of $2 \times 10^{6}$ cells were inoculated subcutaneously into the right dorsal flank. The size of the tumor was measured twice per week using a digital vernier caliper. The tumor volume was determined from the orthogonal dimensions (d1, d2, d3) using the formula $(\mathrm{d} 1 \times \mathrm{d} 2 \mathrm{xd} 3) \times \pi / 6$. Five weeks after inoculation, the mice were euthanized by cervical dislocation, and xenograft tumors were collected.

ELISA. Collected venous blood was centrifuged for $10 \mathrm{~min}$ at 2,000 rpm. The upper tube of serum was then taken for cryopreservation and stored at $-40^{\circ} \mathrm{C}$ for ELISA assay. IGF-1 and IGFBF-3 ELISA kits were obtained from Senxiong Biotech (Shanghai, China). The standard curve was established according to the manufacturer's instructions. Serum samples $(100 \mu \mathrm{l})$ were incubated in each well for $120 \mathrm{~min}$, followed by $50 \mu \mathrm{l}$ primary antibody buffer for $60 \mathrm{~min}, 100 \mu \mathrm{l}$ secondary primary antibody buffer for $60 \mathrm{~min}, 100 \mu \mathrm{l}$ substrate buffer for $10 \mathrm{~min}$ and 1 drop stop solution. Then the plate was read at $490 \mathrm{~nm}$ on the Model 680 microplate reader. The serum concentrations of IGF-1 and IGFBP3 were calculated by the standard curves.

Statistical analysis. All statistical analyses were performed using the SPSS 13.0 package. Pearson's $\chi^{2}$ test was used to detect the expression difference in the cancer and normal tissues. All tests of statistical significance were two-sided, and $\mathrm{P}<0.05$ was considered to indicate a statistically significant difference. All quantitative data are presented as means \pm SD. Independent samples t-test and one-way ANOVA were adopted to compare the means.

\section{Results}

Bioinformatic analysis of IGF-1R in ESCC. In order to study the expression and potential function of IGF-1R in ESCC, we took advantage of publicly available gene expression data (GSE33426 and GSE23400). In the referenced studies, tumor cluster and normal cells separated by laser-capture microscope or tumor tissues and adjacent normal tissues were compared by cDNA microarray. IGF-1R was identified as a differentially expressed gene in both studies. Moreover, we found 85 and 50 genes, respectively, related with the IGF-1R signaling pathway (Fig. 1A and B). Twenty-three genes in the IGF-1R signaling pathway were identified in both studies (Fig. 1C and D). After IPA analysis, we found that these genes were mainly mapped to AKT and MEK cascade and were related to cell growth, proliferation and survival (Fig. 1E). The bioinformatic analysis supported our hypothesis that IGF-1R plays an important role in ESCC.

$I G F-1 R$ is highly expressed in human ESCC cancer tissues. Firstly, we used immunohistochemical (IHC) staining to detect the expression of IGF-1R in ESCC tumor tissues (Fig. 2A and C) and adjacent normal tissues (Fig. 2B and D). After IHC staining, IGF-1R showed strong staining in the cancer cell membrane. Of the 80 cancer specimens, 11 cases were negative, 28 cases were weakly positive and 41 cases were strongly positive. The total positive rate was $86.25 \%$ and the strong positive rate was $51.25 \%$. Of the 18 normal tumor-adjacent tissues, 7 cases were negative, 9 cases were weakly positive and 2 cases were strongly positive. The total positive rate was $61.11 \%$ and the strong positive rate was $11.11 \%$. Both the total positive rate and the strong positive rate were significantly higher in the ESCC tissues than in the normal tumor-adjacent esophageal tissues (total positive rate, $\chi^{2}=4.630, \mathrm{P}<0.05$; strong positive rate, $\chi^{2}=9.614, \mathrm{P}<0.01$ ). In addition, we found a relationship between the expression of IGF-1R and cancer patient characteristics (Table I). We found that high expression of IGF-1R was associated with more aggressive lymph node metastasis, lower histological grade and advanced clinical stage. Both positive and strong positive rates of IGF-1R expression were also higher in patients with 

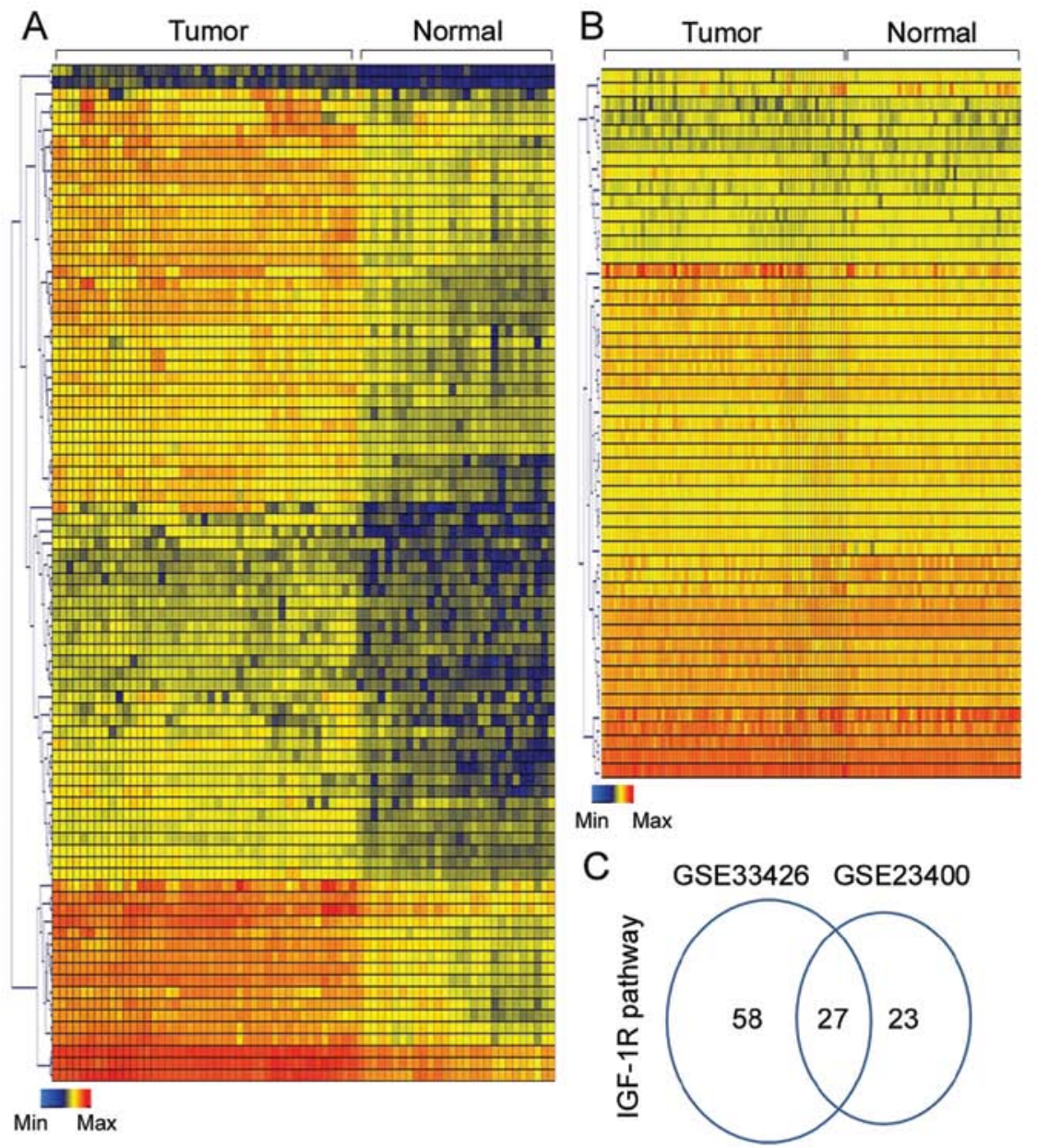

D
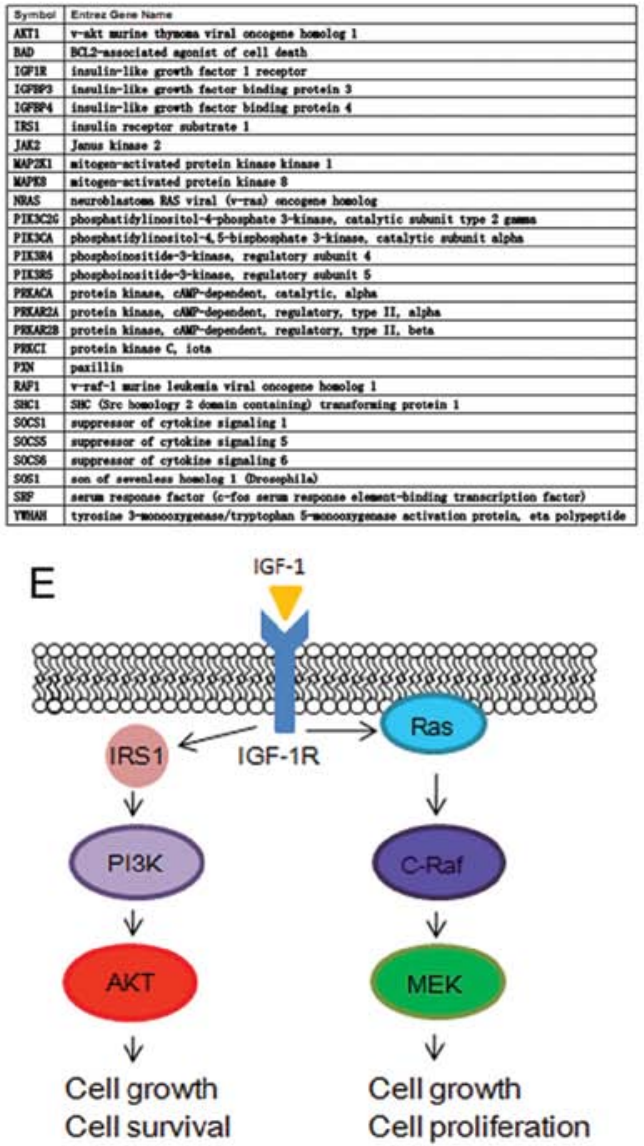

Figure 1. Bioinformatic analysis identifies the upregulation of the IGF-1R signaling pathway components in ESCC. (A) Heat map depicting 83 genes related to the IGF-1R pathway in GSE33426. (B) Heat map depicting 50 genes related to the IGF-1R pathway in GSE22340. (C) Venn diagram showing the common genes identified in GSE33426 and GSE23400. (D) Gene list for the 27 common genes identified in GSE33426 and GSE23400. (E) The core molecular events of the IGF-1R signaling pathway in ESCC.
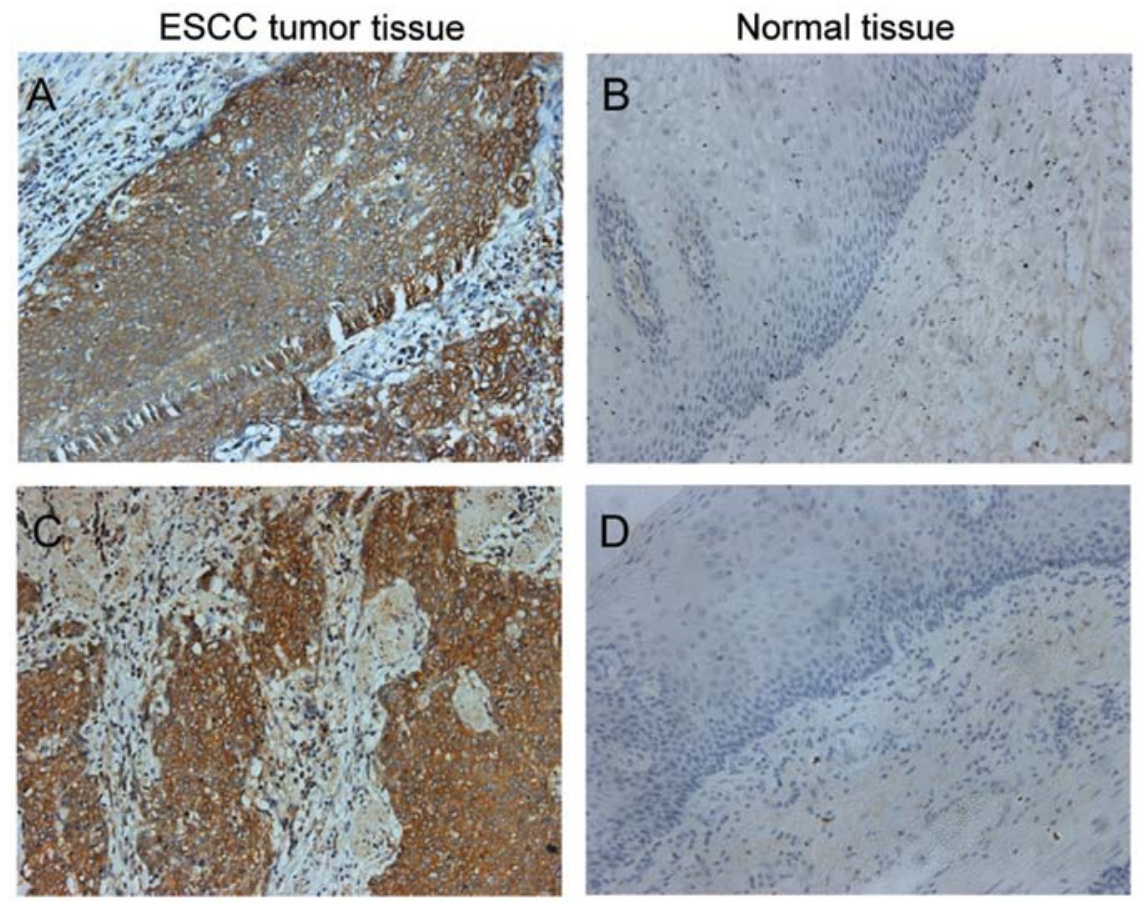

Figure 2. Expression of IGF-1R in ESCC and normal tissues. Tissues in A-D were from the same patients. (A and C) Staining in tumor tissues. (B and D) Staining in adjacent normal tissues. 
Table I. Association of IGF-1R with clinical characteristics.

\begin{tabular}{|c|c|c|c|c|c|}
\hline $\begin{array}{l}\text { Clinical } \\
\text { characteristics }\end{array}$ & $\begin{array}{c}\text { Negative } \\
\mathrm{n}(\%)\end{array}$ & $\begin{array}{c}\text { Weak positive } \\
\text { n }(\%)\end{array}$ & $\begin{array}{c}\text { Strong positive } \\
\mathrm{n}(\%)\end{array}$ & $\begin{array}{c}\text { P-value } \\
\text { (total positive) }\end{array}$ & $\begin{array}{c}\text { P-value } \\
\text { (strong positive) }\end{array}$ \\
\hline \multicolumn{6}{|l|}{ Gender } \\
\hline Female & $8(10.7)$ & $19(32.2)$ & $25(57.2)$ & $>0.05$ & $>0.05$ \\
\hline Male & $3(15.4)$ & $9(36.5)$ & $16(48.1)$ & & \\
\hline \multicolumn{6}{|l|}{ Age, years } \\
\hline$<51$ & $1 \quad(7.7)$ & $5(38.5)$ & $7(53.8)$ & $>0.05$ & $>0.05$ \\
\hline $51-65$ & $5(11.6)$ & $18(38.3)$ & $24(51.1)$ & & \\
\hline$>65$ & $5(25.0)$ & $5(25.0)$ & $10(50.0)$ & & \\
\hline \multicolumn{6}{|c|}{ Lymph node metastasis } \\
\hline Yes & $3(5.6)$ & $11(20.3)$ & $40(74.1)$ & $<0.05$ & $<0.05$ \\
\hline No & $8(30.8)$ & $17(65.3)$ & $1 \quad(3.9)$ & & \\
\hline \multicolumn{6}{|c|}{ Histological grade } \\
\hline Low & $1 \quad(4.8)$ & $3(14.2)$ & $17(81.0)$ & $<0.05$ & $<0.05$ \\
\hline Moderate & $3(8.1)$ & $16(43.2)$ & $18(48.7)$ & & \\
\hline High & $7(30.2)$ & $9(40.9)$ & $6(27.3)$ & & \\
\hline \multicolumn{6}{|l|}{ Clinical stage } \\
\hline I-II & $10(24.7)$ & $18(43.9)$ & $13(31.7)$ & $<0.01$ & $<0.01$ \\
\hline III-IV & $1 \quad(2.6)$ & $10(25.6)$ & $28(71.8)$ & & \\
\hline
\end{tabular}

lymph node metastasis than those without lymph node metastasis $(\mathrm{P}<0.01)$, and were also higher in patients with advanced clinical stage (III-IV stage) than those with early clinical stage (I-II stage). The expression of IGF-1R was also increased from a low differentiation grade to a moderate differentiation stage and high differentiation grade $(\mathrm{P}<0.05$ between each of the two groups). No significant difference was noted in relation to age or gender $(\mathrm{P}>0.05)$. These results showed that IGF-1R was highly expressed in ESCC tissues, and the high expression of IGF-1R in the ESCC tissues was related to tumor progression.

$I G F-1 R$ acts as an oncogene in ESCC in vitro and in vivo. We determined the expression of IGF-1R in EC9706, EC109 and NEC cells and found that IGF-1R was relatively highly expressed in these three ESCC cell lines. We established stable IGF-1R-knockdown cells by transfection of pGCsilencer ${ }^{\text {TM U6/ }}$ Neo/GFP/RNAi IGF-1R in EC9706 cells. Western blotting and RT-PCR verified the successful knockdown of IGF-1R in these cells (Fig. 3A and B). Compared with the empty vector control, the cell proliferation was inhibited and the doubling time was extended significantly in the IGF-1R-knockdown cells (Fig. 3C). Significant cell cycle arrest occured in the IGF-1R-knockdown cells with an increased percentage of $\mathrm{G} 1$ phase cells $(\mathrm{P}<0.05)$ and a decreased percentage of $\mathrm{S}$ phase cells $(\mathrm{P}<0.01)$ (Fig. 3D). In addition, a higher apoptosis rate $(\mathrm{P}<0.05)$ and weaker clonogenesis ability $(\mathrm{P}<0.05)$ were found after IGF-IR-knockdown (Fig. 3E). All of these results showed that IGF-1R acts as an oncogene in ESCC in vitro. We also used a mouse ESCC xenograft model to detect the tumor growth ability of IGF-1R in vivo. Ten days after cell inoculation, subcutaneous nodules were noted and finally developed into tumors. Although all three cell lines induced visible tumors in the nude mouse, the size of the tumors induced by the stable IGF-1R-knockdown cells was significantly smaller than those formed by the empty control $(\mathrm{P}<0.05)$ and wild-type cells $(\mathrm{P}<0.05)$ (Fig. 4A and $\mathrm{B})$. The animal assay showed that IGF-1R can induce tumor growth in vivo.

High expression of $I G F-1 R$ is related to low sensitivity to chemotherapy in the ESCC cell lines. We compared the sensitivity to 5-FU and cisplatin between the IGF-1Rknockdown cells and the empty vector control. We found that the growth inhibition rate of 5-FU and cisplatin in the pGCsilencer ${ }^{\mathrm{TM}}$ U6-IGF-1R-transfected cells was higher than that in the empty vector-transfected and the non-transfected cells $(\mathrm{P}<0.05)$ (Fig. 5A). The high expression of IGF-1R facilitates cancer cell resistance to chemotherapy.

Serum concentrations of IGF-1/IGFBP-3 are associated with the chemotherapy response. We determined the serum concentrations of IGF-1 and IGFBP-3 in 172 patients both before and after chemotherapy treatment (Fig. 5B). The mean serum concentrations of IGF-1 pre-therapy and post-therapy in all patients were $268.87 \pm 61.66$ and $266.42 \pm 49.98 \mathrm{ng} / \mathrm{ml}$, respectively, without significant difference. The mean serum concentrations of IGFBP-3 pre-therapy and post-therapy in all patients were $2523.2 \pm 469.83$ and $2598.8 \pm 563.56 \mathrm{ng} / \mathrm{ml}$, respectively, without significant difference. In contrast, the serum concentration of IGFBP-3 increased $(\mathrm{P}<0.01)$ and the IGF-1/IGFBP-3 ratio decreased $(\mathrm{P}=0.01)$ significantly after chemotherapy in the chemotherapy responsive group (CR and PR). The serum concentration of IGF-1 decreased after chemotherapy in the responsive groups but without significance $(\mathrm{P}>0.05)$. Accordingly, in the chemotherapy unresponsive group (SD and PD) the IGF-1/IGFBP-3 ratio increased 


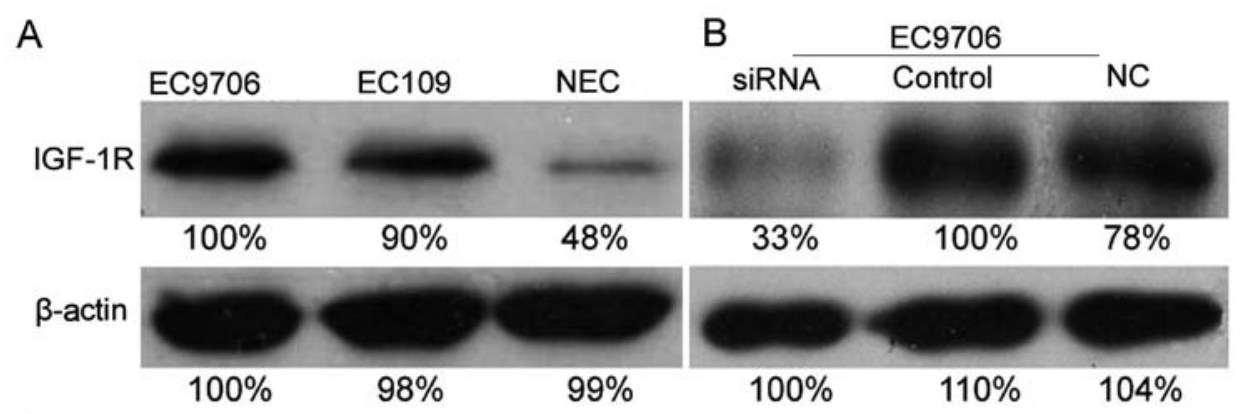

C

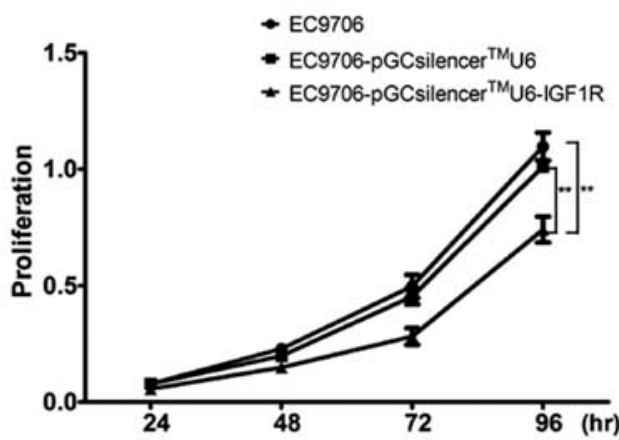

D

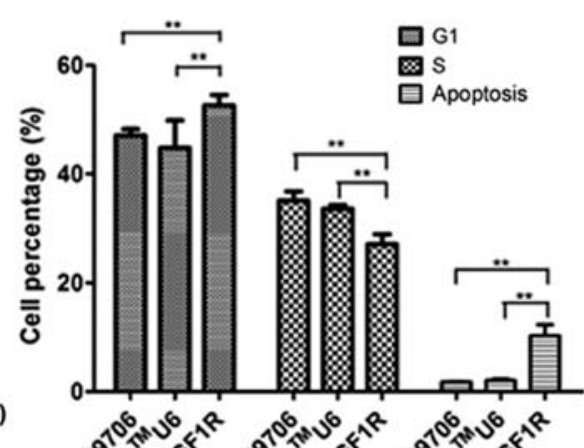

$E$
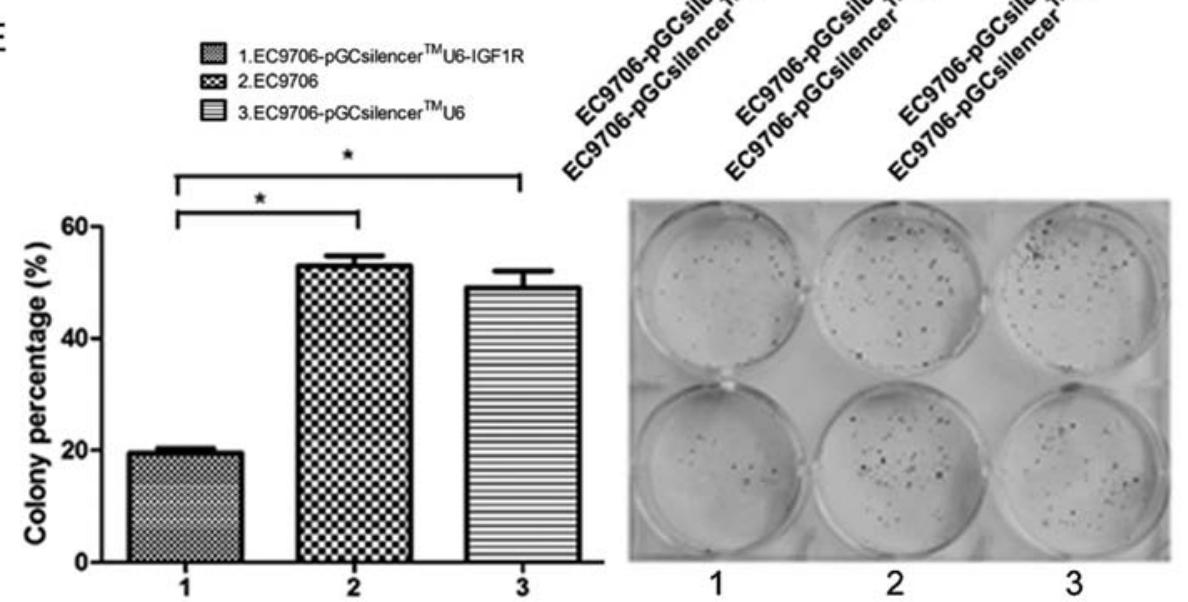

Figure 3. Biological function of IGF-1R in the ESCC cell lines. (A) IGF-1R protein expression in three ESCC cell lines. (B) Expression of IGF-1R in the EC9706 cells after pGCsilencer ${ }^{\mathrm{TM}} \mathrm{U6} / \mathrm{Neo} / \mathrm{GFP} / \mathrm{RNAi}-\mathrm{IGF}-1 \mathrm{R}$ transfection (siRNA) or without any treatment (Control) or pGCsilencer ${ }^{\mathrm{TM}} \mathrm{U6} / \mathrm{Neo} / \mathrm{GFP}$ empty plasmid (NC). (C) Growth curve of cells after different treatments. (D) Cell cycle and apoptosis analysis for cells after different treatment. (E) Colony formation abilities of cells after different treatments. ${ }^{*} \mathrm{P}<0.05,{ }^{* *} \mathrm{P}<0.01$.

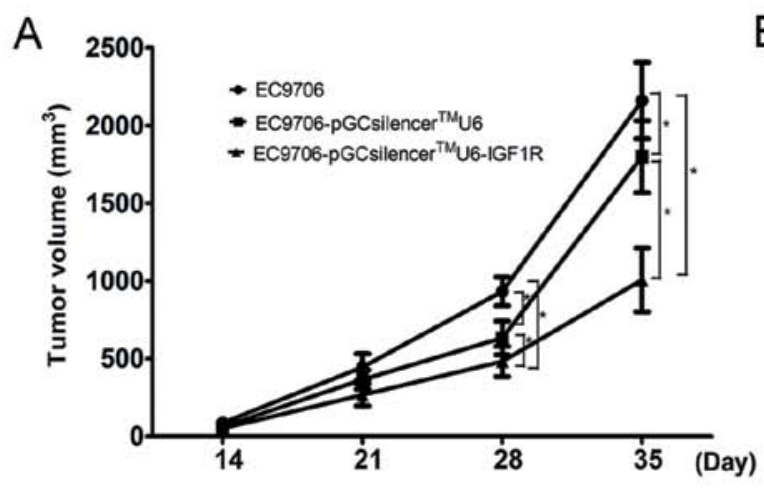

1 EC9706

2 EC9706-pGCsilencer ${ }^{\mathrm{TM} U 6}$

3 EC9706-pGCsilencer ${ }^{\mathrm{TM} U 6-I G F 1 R}$

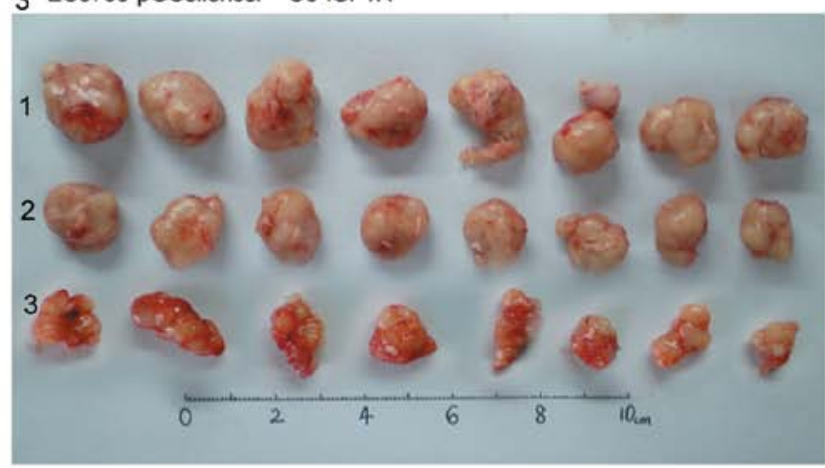

Figure 4. IGF-1R induces tumor growth in mouse xenografts. (A) The volume of tumors in the different groups. (B) The size of tumors in the different groups. ${ }^{*} \mathrm{P}<0.05$ 


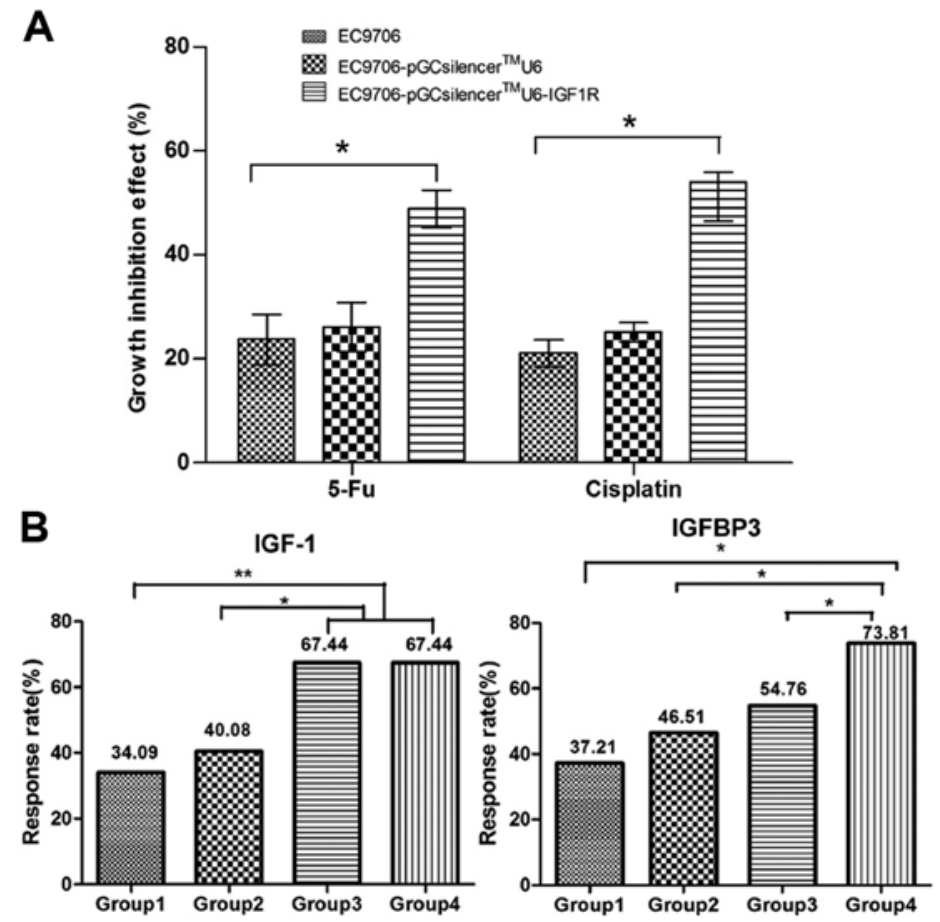

Figure 5. IGF-1R is associated with chemotherapeutic response in ESCC. (A) Cells following knockdown of IGF-1R showed more sensitive to 5-FU and cisplatin. (B) Patients with higher expression of IGF1 and IGFBP-3 showed higher rates of chemotherapeutic response.

Table II. Serum concentration of IGF-1, IGFBP-3 and IGF-1/IGFBP-3 ratio.

\begin{tabular}{lcccc}
\hline Groups & $\mathrm{n}$ & IGF-1 $(\mathrm{ng} / \mathrm{ml})$ & IGFBP-3 $(\mathrm{ng} / \mathrm{ml})$ & IGF-1/IGFBP-3 \\
\hline Chemotherapy responsive & & & & \\
Pre-therapy & 90 & $284.5 \pm 56.5$ & $2599.4 \pm 444.6$ & $0.11391 \pm 0.034$ \\
Post-therapy & 90 & $270.0 \pm 50.1$ & $2854.3 \pm 559.0$ & $0.10030 \pm 0.036$ \\
P-value & & 0.07 & 0.001 & 0.01 \\
Chemotherapy unresponsive & & & & \\
Pre-therapy & 82 & $251.7 \pm 62.8$ & $2439.5 \pm 485.1$ & $0.10723 \pm 0.034$ \\
Post-therapy & 82 & $262.5 \pm 49.9$ & $2318.5 \pm 418.4$ & $0.11820 \pm 0.035$ \\
P-value & & 0.226 & 0.089 & 0.045 \\
\end{tabular}

significantly $(\mathrm{P}<0.05)$ after chemotherapy; the concentration of IGFBP-3 decreased but without significance $(\mathrm{P}>0.05)$. There was no variation in IGF-1 (Table II). These results showed that the IGF-1/IGFBP-3 ratio can be used to predict the chemotherapeutic effect; a decreased IGF-1/IGFBP-3 molar ratio presents a better chemotherapeutic effect. We also evaluated the chemotherapy response rate in 4 groups divided according to the quartiles of plasma IGF-1 and IGFBP-3. Chemotherapy response rate increased from low concentrations of IGF-1 and IGFBP-3 to high concentrations of IGF-1 and IGFBP-3. These results showed that increased serum levels of IGF-1 and IGFBP-3 are associated with significantly higher rates of tumor response $(\mathrm{P}<0.05)$.

\section{Discussion}

In the present study, we determined the expression of IGF-1R in an ESCC cohort and verified the high expression of IGF-1R in ESCC tumor tissues when compared with that in adjacent normal tissues. High expression of IGF-1R was found to be associated with aggressive lymph node metastasis, lower histological grade and advanced clinical stage. After knockdown of IGF-1R in ESCC cell lines and the mouse xenograft assay, we verified the pro-proliferation, apoptosis inhibition and tumor growth function of IGF-1R in vitro and in vivo. We also found that IGF-1R was associated with the response to standard chemotherapy drugs 5-FU and cisplatin in the ESCC cell lines. More importantly, we found that the serum concentration of IGF-1/IGFBP3 can be used for the predicting chemotherapeutic effect. Increased serum levels of IGF-1 and IGFBP-3 were found to be associated with significantly higher rates of tumor response.

High expression of IGF-1R has been reported in several cancers including ESCC. Imsumran et al used IHC to detect the expression of IGF-1R in ESCC tumor tissues and found that $\sim 50 \%$ of tissues showed high expression of IGF-1R which 
was associated with invasive depth, metastasis, advanced tumor stage and recurrence (22). In this study, we also detected high expression of IGF-1R in ESCC tissues compared with that in adjacent normal tissues. We found that high expression of IGF-1R was associated with more aggressive lymph node metastasis, lower histological grade and advanced clinical stage, similar to the results of previous research. We suggest that the expression of IGF-1R can be used as a biomarker for the diagnosis and tumor progression of ESCC.

IGF-1R plays an important role in carcinogenesis mainly based on the activation of the PI3K/AKT/mTOR signaling and the Ras/Raf/MEK/MAPK pathways. The activation of AKT and MAPK pathways help cancer cells acquire the ability for proliferation, evasion of apoptosis, insensitivity to antigrowth signals, unlimited replicative potential, metastasis and angiogenesis $(24,25)$. Both of these pathways are reported to be activated in ESCC. After using siRNA to knock down the expression of IGF-1R, we found significant inhibition of cell growth, cell cycle arrest, reduced apoptosis and fewer colonies. These results are the same as previous findings. Various strategies such as anti-IGF-1R antibodies, IGF-1 mimetic peptides, antisense strategies, IGF-1R-specific peptide aptamers, targeted degradation of IGF-1R and expression of dominantnegative IGF-1R mutants have been explored to inhibit IGF-1R signaling (26). AVE1642, a humanized version of the murine monoclonal antibody can bind specifically and with high affinity to human IGF-1R preventing IGF-1R binding to its ligand resulting in receptor inactivation (27). It has been reported to delay the growth of tumor xenografts and to prolong the survival of tumor-bearing nude mice. An IGF-1R inhibitor NVP-AEW541 showed significant inhibition of pancreatic cell lines, and is being used for an in vivo study (28). Another small molecular IGF-1R inhibitor BMS-554417 showed antitumor activity in breast cancer in vitro and in vivo (29). The small molecular inhibitor for IGF-1R and IGF-1R antibodies both can be used for ESCC treatment (30). In further research, we will utilize the IGF-1R antibody or a small molecular inhibitor for IGF-1R to treat cells or animals to verify new strategies for the targeted therapy for IGF-1R.

Chemotherapy resistance is a serious obstacle to cancer therapy. Any cellular and molecular events associated with chemotherapeutic effects are possible targets for better outcomes from treatment. We found that high expression of IGF-1R facilitated ESCC cell resistance to chemotherapy. Liu et al reported that IGF-I prevented the apoptosis in ESCC CE81T/VGH cells induced by chemotherapeutic drugs, such as cisplatin, 5-FU and camptothecin (23). Thus, interruption of IGF-IR function may provide a strategy by which to retard tumor growth and increase the sensitivity of esophageal carcinoma to chemotherapy. Due to the important bioactivities of the ligand for IGF-1R, IGF1, we hypothesized that chemotherapy may influence the serum levels of IGF-1 and IGFBP-3. In order to prove this hypothesis, we compared the serum concentrations of IGF-1 and IGFBP3 in ESCC patients before and after chemotherapy. Our results showed that the IGF-1/IGFBP-3 ratio was significantly decrease after chemotherapy in the chemotherapy responsive group (CR and $\mathrm{PR}$ ) and was increased in the chemotherapy unresponsive group (SD and PD). The IGF-1/IGFBP3 ratio can be used to predict the chemotherapeutic effect. The serum concentration of IGFBP3 was increased in the chemotherapy responsive group and had a tendency to decrease in the chemotherapy unresponsive group. We divided patients into 4 groups according to quartiles of serum IGF-1 and IGFBP-3 and then compared the chemotherapy response rate in these 4 groups. We found that the chemotherapy response rate increased from low concentrations of IGF-1 and IGFBP-3 to high concentrations of IGF-1 and IGFBP-3. These results showed that increasing serum IGF-1 and IGFBP-3 are associated with significantly higher rates of tumor response $(\mathrm{P}<0.05)$. Since almost $90 \%$ of circulating IGFs bind with IGFBP-3, we believe that the concentration of IGF-1/IGFBP-3 is more effective for predicting the chemotherapeutic effect. We believe that the potential function of IGF-1/IGFBP-3 to predict chemotherapeutic effect is also related to the function of IGF-1R.

In conclusion, in the present study we verified the oncogenic function of IGF-1R in vitro and in vivo. We proved that a high serum concentration of IGF-1R is associated with chemotherapy resistance. This study offers strong evidence for the application of IGF-1R as a new target for ESCC therapy.

\section{Acknowledgements}

We thank all the patients who participated in this study. We thank Dr Jane Yu for the suggestions concerning the bioinformatic analysis.

\section{References}

1. Jemal A, Bray F, Center MM, et al: Global cancer statistics. CA Cancer J Clin 61: 69-90, 2011.

2. He J, Gu D, Wu X, et al: Major causes of death among men and women in China. N Engl J Med 353: 1124-1134, 2005.

3. Stoner GD and Gupta A: Etiology and chemoprevention of esophageal squamous cell carcinoma. Carcinogenesis 22: 1737-1746, 2001.

4. Qi YJ, Chao WX and Chiu JF: An overview of esophageal squamous cell carcinoma proteomics. J Proteomics 75: 3129-3137, 2012.

5. Holmes RS and Vaughan TL: Epidemiology and pathogenesis of esophageal cancer. Semin Radiat Oncol 17: 2-9, 2007.

6. Lin Q, Gao XS, Qiao XY, et al: Phase I trial of escalating-dose cisplatin with 5-fluorouracil and concurrent radiotherapy in Chinese patients with esophageal cancer. Acta Med Okayama 62: 37-44, 2008.

7. Liao Y, Abel U, Grobholz R, et al: Up-regulation of insulin-like growth factor axis components in human primary prostate cancer correlates with tumor grade. Hum Pathol 36: 1186-1196, 2005.

8. Tsuta K, Mimae T, Nitta H, et al: Insulin-like growth factor-1 receptor protein expression and gene copy number alterations in non-small cell lung carcinomas. Hum Pathol 44: 975-982, 2013.

9. Rikhof B, de Jong S, Suurmeijer AJ, Meijer C and van der Graaf WT: The insulin-like growth factor system and sarcomas. J Pathol 217: 469-482, 2009.

10. Ucar DA, Magis AT, He DH, et al: Inhibiting the interaction of cMET and IGF-1R with FAK effectively reduces growth of pancreatic cancer cells in vitro and in vivo. Anticancer Agents Med Chem 13: 595-602, 2013.

11. Jia Y, Zhang Y, Qiao C, et al: IGF-1R and ErbB3/HER3 contribute to enhanced proliferation and carcinogenesis in trastuzumab-resistant ovarian cancer model. Biochem Biophys Res Commun 436: 740-745, 2013.

12. Sharmila G, Bhat FA, Arunkumar R, et al: Chemopreventive effect of quercetin, a natural dietary flavonoid on prostate cancer in in vivo model. Clin Nutr: Sep 3, 2013 (Epub ahead of print). doi: 10.1016/j.clnu.2013.08.011.

13. Wilson S and Chia SK: IGF-1R inhibition: right direction, wrong pathway? Lancet Oncol 14: 182-183, 2013. 
14. Zhao X, Dou W, He L, et al: MicroRNA-7 functions as an anti-metastatic microRNA in gastric cancer by targeting insulin-like growth factor-1 receptor. Oncogene 32: 1363-1372, 2013.

15. Werner $\mathrm{H}$ and Bruchim I: The insulin-like growth factor-I receptor as an oncogene. Arch Physiol Biochem 115: 58-71, 2009.

16. Wei Z, Hurtt R, Gu T, et al: GRK2 negatively regulates IGF-1R signaling pathway and cyclins' expression in HepG2 cells. J Cell Physiol 228: 1897-1901, 2013.

17. Fürstenberger G, Senn E, Morant R, Bolliger B and Senn HJ: Serum levels of IGF-1 and IGFBP-3 during adjuvant chemotherapy for primary breast cancer. Breast 15: 64-68, 2006.

18. Ozkan EE: Plasma and tissue insulin-like growth factor-I receptor (IGF-IR) as a prognostic marker for prostate cancer and anti-IGF-IR agents as novel therapeutic strategy for refractory cases: a review. Mol Cell Endocrinol 344: 1-24, 2011.

19. Thariat J, Bensadoun RJ, Etienne-Grimaldi MC, et al: Contrasted outcomes to gefitinib on tumoral IGF1R expression in head and neck cancer patients receiving postoperative chemoradiation (GORTEC trial 2004-02). Clin Cancer Res 18: 5123-5133, 2012

20. Peled N, Wynes MW, Ikeda N, et al: Insulin-like growth factor-1 receptor (IGF-1R) as a biomarker for resistance to the tyrosine kinase inhibitor gefitinib in non-small cell lung cancer. Cell Oncol 36: 277-288, 2013

21. Jones HE, Gee JM, Barrow D, et al: Inhibition of insulin receptor isoform-A signalling restores sensitivity to gefitinib in previously de novo resistant colon cancer cells. Br J Cancer 95: 172-180, 2006.

22. Imsumran A, Adachi Y, Yamamoto $\mathrm{H}$, et al: Insulin-like growth factor-I receptor as a marker for prognosis and a therapeutic target in human esophageal squamous cell carcinoma. Carcinogenesis 28: 947-956, 2007.
23. Liu YC, Leu CM, Wong FH, et al: Autocrine stimulation by insulin-like growth factor I is involved in the growth, tumorigenicity and chemoresistance of human esophageal carcinoma cells. J Biomed Sci 9: 665-674, 2002.

24. Dhillon AS, Hagan S, Rath O and Kolch W: MAP kinase signalling pathways in cancer. Oncogene 26: 3279-3290, 2007.

25. Hanahan D and Weinberg RA: Hallmarks of cancer: the next generation. Cell 144: 646-674, 2011.

26. Negi A, Ramarao P and Kumar R: Recent advancements in small molecule inhibitors of insulin-like growth factor- 1 receptor (IGF-1R) tyrosine kinase as anticancer agents. Mini Rev Med Chem 13: 653-681, 2013.

27. Soria JC, Massard C, Lazar V, et al: A dose finding, safety and pharmacokinetic study of AVE1642, an anti-insulin-like growth factor-1 receptor (IGF-1R/CD221) monoclonal antibody, administered as a single agent and in combination with docetaxel in patients with advanced solid tumours. Eur J Cancer 49: 1799-1807, 2013.

28. Ioannou N, Seddon AM, Dalgleish A, Mackintosh D and Modjtahedi H: Treatment with a combination of the ErbB (HER) family blocker afatinib and the IGF-IR inhibitor, NVP-AEW541 induces synergistic growth inhibition of human pancreatic cancer cells. BMC Cancer 13: 41, 2013.

29. Haluska P, Carboni JM, Loegering DA, et al: In vitro and in vivo antitumor effects of the dual insulin-like growth factor-I/insulin receptor inhibitor, BMS-554417. Cancer Res 66: 362-371, 2006.

30. Ucar DA, Cox A, He DH, et al: A novel small molecule inhibitor of FAK and IGF-1R protein interactions decreases growth of human esophageal carcinoma. Anticancer Agents Med Chem 11: 629-637, 2011. 